Polymer Journal, Vol. 38, No. 8, pp. 781-785 (2006)

(C) 2006 The Society of Polymer Science, Japan

\title{
Significant Effect of Dopant Size on Nanoscale Fractal Structure of Polypyrrole Film
}

\author{
Ali Eftekhari, ${ }^{\dagger}$ Mahmood KazemZad, and Mansoor Keyanpour-RAD
}

Laboratory of Electrochemistry, Materials and Energy Research Center, P.O. Box 14155-4777, Tehran, Iran

(Received November 16, 2005; Accepted April 1, 2006; Published June 30, 2006)

\begin{abstract}
Surface morphologies of thin films of polypyrrole doped with two similar anions (with different ionic radii) viz. chloride and bromide were compared by means of fractal geometry. Scanning electron micrographs of the polymer surfaces showed a significant difference between the surface structures of two anion-doped polypyrrole films in microscale and particularly in nanoscale. Surprisingly, the bromide-doped polypyrrole had nanostructure; whereas, the chloride-doped polypyrrole surface was smooth in nanoscale. An electrochemical method based on gold-masking approach was employed to reveal the fractality of these polymer surfaces in microscale. Fractal geometry successfully discovered the effect of anion doping on microstructure of the conductive polymer by suggesting different fractal dimensions. In addition, SAXS analysis indicated significant difference in fractality of the polymer in nanoscale. [doi:10.1295/polymj.PJ2005173]

KEY WORDS Surface Morphology / Nanoscale Roughness / Fractal / Conductive Polymer / Nanostructures / Electrode Surface /
\end{abstract}

It is well known that anion doping has a significant influence on surface morphology of conductive polymers. As anion doping is an essential process in the course of polymer synthesis, this issue is of great interest. ${ }^{1-12}$ There are two affecting factors in anion doping: size of the anion incorporated into the polymer structure and the synthesis mechanism in the presence of the anion. To inspect the second factor, it is only needed to choose two different anions with approximately the same ionic radii. However, it is difficult to investigate the first factor since anion effects on the polymerization are essentially different. Investigations in this context are mainly restricted to common anions such as $\mathrm{Cl}^{-}, \mathrm{NO}_{3}{ }^{-}, \mathrm{ClO}_{4}{ }^{-}, \mathrm{SO}_{4}{ }^{2-}$, etc. ${ }^{1-12}$ As Compton and coworkers emphasized the inner oxygen bond in the anion has a significant effect on the polymerization process. ${ }^{8}$ Thus, comparison of monoatomic anions such as $\mathrm{Cl}^{-}$with other anions is not correct. To overcome this problem, we aim to compare two similar monoatomic anions as dopants, since this strategy is not usually followed in the literature.

Fractal geometry as a powerful tool was employed for surface analysis of a typical conductive polymer. Applicability of fractal analysis for surface studies of conductive polymers has been widely reported in the literature. ${ }^{13-21}$ Although, it has been emphasized that usefulness of fractal analysis is related to comparative studies of similar surfaces, less attention has been paid to this issue for the investigation of surface morphology of different anion-doped conductive polymers. Gobal et al. have investigated the fractal structure of anion-doped poly-ortho-aminophenol films by means of electrochemical methods, and concluded that anion doping has no effect on fractal structure of conductive polymers. ${ }^{22}$ This incorrect conclusion (as it is well known that surface morphology of a conductive polymer is highly dependent on doping process) is due to the methodology misusing, as described elsewhere. ${ }^{23}$ Fractal analysis by means of electrochemical techniques (such as cyclic voltammetry and chronoamperometry) requires the process of 'diffusion toward electrode surface' to sense fractal structure of the electrode surface; whereas, electrochemical redox systems of conductive polymers are not merely a surface reaction, but mainly solid-state diffusion. Here, we show that upon appropriate utilization of electrochemical methods, they can be successfully applied to reveal difference in fractal structures of anion-doped polymer surfaces.

\section{EXPERIMENTAL}

Polypyrrole as a well-known conductive polymer was typically chosen for this study. Thin films of polypyrrole were conventionally synthesized in the presence of two anions viz. chloride and bromide. The conductive polymer was electrochemically deposited onto a Pt substrate surface from acidic aqueous solutions. The electrolyte solutions were prepared from $0.030 \mathrm{~mol} / \mathrm{L}$ of the monomer in $0.5 \mathrm{~mol} / \mathrm{L}$ of the corresponding acid ( $\mathrm{HCl}$ or $\mathrm{HBr})$. Electropolymerization was performed potentiodynamically by potential cycling between -0.2 and $1.0 \mathrm{~V} v s$. $\mathrm{Ag} / \mathrm{AgCl}$ with

${ }^{\dagger}$ To whom correspondence should be addressed (Tel: +98-261-621-0009, Fax: +98-261-620-1888, E-mail: eftekhari@merc.ac.ir). 
AI)
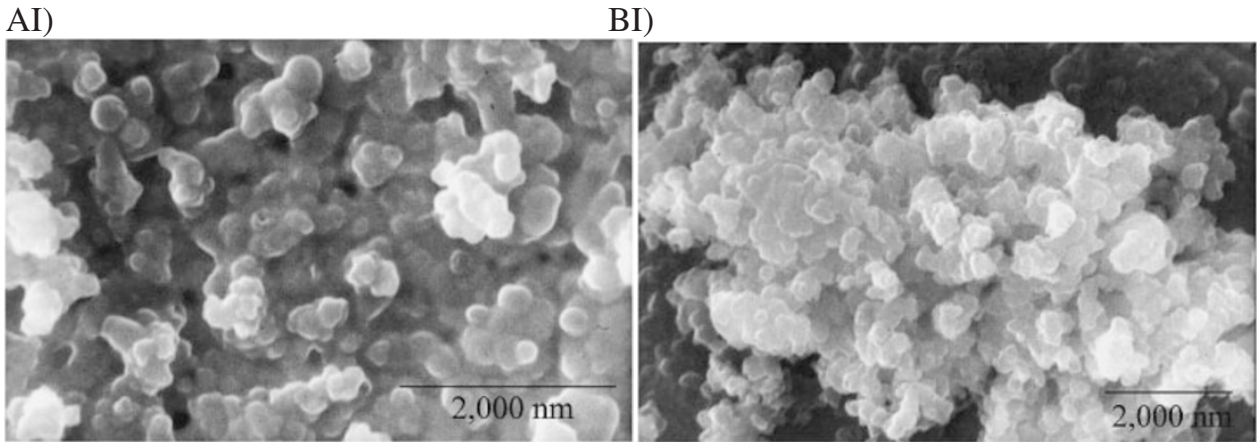

AII)

BII)
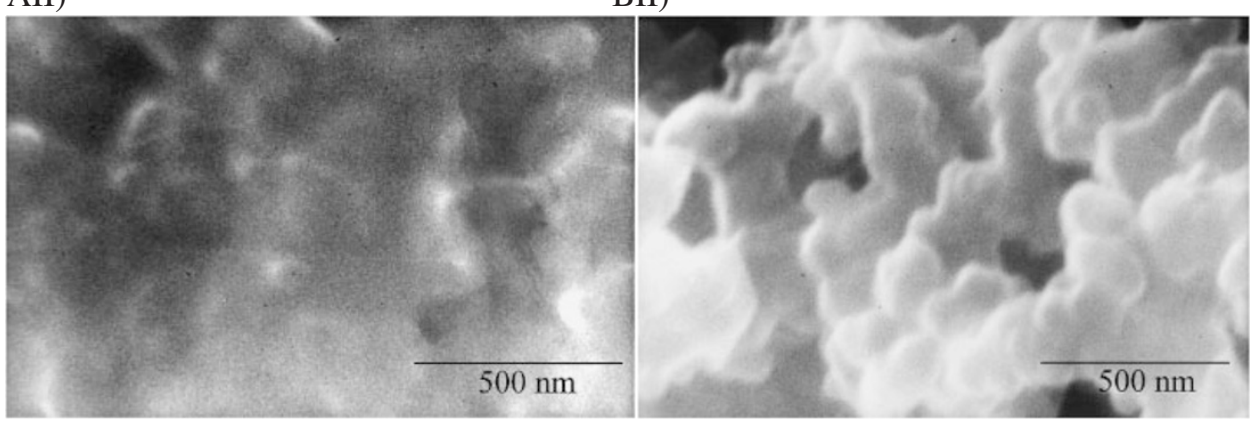

Figure 1. SEM images of (A) chloride- and (B) bromide-doped polypyrrole films electrodeposited onto Pt substrate surface.

scan rate $50 \mathrm{mV} / \mathrm{s}$ for 9 successive cycles. Cyclic voltammetric measurements were performed using a Metrohm 746VA potentiostat.

Fractal analysis of the polymer surfaces was performed by means of cyclic voltammetry. This method is based on the concept of 'diffusion toward electrode surfaces'.${ }^{24}$ Gobal et al. have used the diffusion of the own anions in the course of insertion/extraction into the polymer structure for this purpose..$^{22}$ However, this method is not reliable, since the material surface is subject of severe structural change during the insertion/extraction process. ${ }^{25}$ On the other hand, this is accompanied by side-processes leading to experimental errors. ${ }^{23}$ The best redox system for fractal analysis is the $\mathrm{Fe}(\mathrm{CN})_{6}{ }^{3-} / \mathrm{Fe}(\mathrm{CN})_{6}{ }^{4-}$ system, as preliminarily introduced by Pajkossy and Nyikos. ${ }^{26}$ The electrolyte solution was $5 \mathrm{mM} \mathrm{K} \mathrm{K}_{3} \mathrm{Fe}(\mathrm{CN})_{6}$ in $1.0 \mathrm{M} \mathrm{KCl}$ as supporting electrolyte.

Although, this redox system can be directly investigated on the polymer surface, ${ }^{19}$ there are still sources of experimental errors due to side-reaction such as incorporation of hexacyanoferrate ions into the polymer matrix. To overcome this problem, gold-masking approach has been proposed to generalize the electrochemical methods for a vast variety of solid surfaces ${ }^{27,28}$ and even liquid | liquid interfaces. ${ }^{29}$ To this aim, a thin film of gold was deposited onto the polymer surfaces. This action was also performed for scanning electron microscopy (SEM) imaging, as this technique needs samples with good electrical conductivity. For materials with poor conductivity, it is necessary to coat the sample with a thin layer of gold to meet the method requirement. In spite of relatively good electrical conductivity, conductive polymers normally fall in this class of materials in SEM imaging. SEM images were recorded using a Cambridge electron microscope model Steroscan 360 at operating voltage of $20 \mathrm{kV}$. Small-angle X-ray scattering (SAXS) measurements were performed by means of a PW 3710 Philips system employing a rotating X-ray generator under appropriate conditions.

\section{RESULTS AND DISCUSSION}

The most common technique for inspecting the surface morphology is SEM. The results obtained from SEM imaging of the anion-doped polypyrrole films obviously display the significant difference between their surface structures (Figure 1). It seems that chloride doping causes the formation of polymer particles spread across the substrate surface (Figure 1AI). Whereas, particles of the bromide-doped polypyrrole tend to form dense clusters, as agglomeration of individual particles is accompanied by close packaging in this case (Figure 1BI). This is indeed the microscale surface morphology for anion-doped polypyrrole films. Whereas, surface structure of the anion-doped polypyrrole films in nanoscale is of particular interest.

According to Figure 1AII, surface of the chloridedoped polypyrrole film is approximately smooth in nanoscale. Interestingly, the bromide-doped polypyrrole film has a complicated morphology in the same 
scale (Figure 1BII). This suggests that not only anion doping has a significant effect on the surface morphology of a conductive polymer, but also this effect is strongly scale-dependent. Thus, a careful attention should be paid to this issue. Although, it is well known that the nature of dopant has an effect on the morphology of a conductive polymer, however, the difference observed in the present case (particularly in nanoscale) is extremely significant.

To compare surface morphologies of the aniondoped polypyrrole films, fractal geometry provides a quantitative measure namely fractal dimension. Fractal analysis based on electrochemical method can be simply employed for this purpose. As stated above, diffusion of electroactive species as a result of original redox system of the electroactive material is not a reliable approach to investigate fractality of electrode surfaces. ${ }^{25}$ Once again, we follow the great opportunity provided by gold-masking approach ${ }^{25,27-29}$ to achieve reliable results for comparative study of the fractal structures.

To this aim, a thin film of gold with thickness of $c a$. $20 \mathrm{~nm}$ is deposited on the sample surface via a sputtering process. This action was performed to record the SEM images illustrated in Figure 1. Therefore, for fractal analysis of the anion-doped polypyrrole films by means of electrochemical methods using goldmasking approach, it is only needed to use the samples prepared for SEM investigations. Such samples are generally available in this type of investigations, as SEM is an essential technique in studies of surface morphology.

Estimating the fractal dimension of an electrode surface is very easy by means of electrochemical methods. The most common technique for this purpose is cyclic voltammetry. In this case, scan rate dependency of the peak current is employed to inspect the electrochemical response of the system to the diffusion layer thickness, which acts as yardstick length. In other words, we record the electrochemical signal (i.e., representative of the diffusion process) at various yardstick lengths by changing the scan rate. Therefore, it is only needed to record cyclic voltammograms at different scan rates. According to Stromme et al.,${ }^{30}$ by plotting the peak current against the scan rate in a log-log scale, the curve slope indicates the fractal parameter, which is transformed to the fractal dimension with the following relation: $\alpha=\left(D_{\mathrm{f}}-1\right) / 2$.

Figure 2 shows the scan rate dependency of the peak current for both anion-doped polypyrrole films with respect to the 'diffusion toward electrode surfaces' provided by the redox system described in the Experimental Section, as a single diffusing ion is employed for both cases to avoid possible experimental

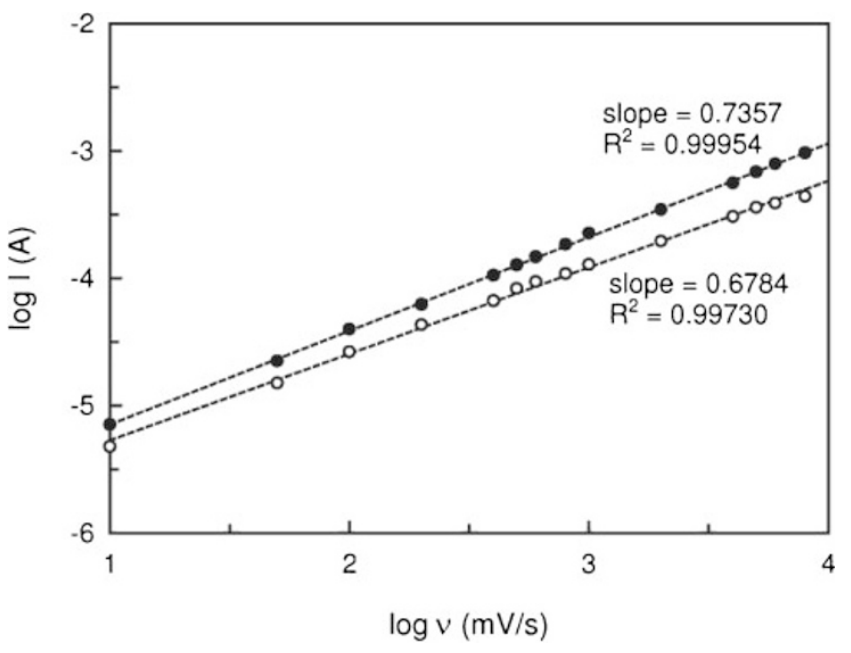

Figure 2. Scan rate dependencies of the peak current $\left(\log I_{\mathrm{p}}-\right.$ $\log v$ ) for the cases of chloride- $(\bigcirc)$ and bromide-doped $(\bullet)$ polypyrrole films. The process of 'diffusion toward electrode surfaces' was provided by the $\mathrm{Fe}(\mathrm{CN})_{6}{ }^{3-} / \mathrm{Fe}(\mathrm{CN})_{6}{ }^{4-}$ redox system. The peak currents recorded correspond to the oxidation of $\mathrm{Fe}(\mathrm{CN})_{6}{ }^{4-}$ ions.

errors associated with difference in the diffusion process. The fractal dimensions estimated from the slopes of corresponding scan rate dependencies were 2.36 and 2.47 for the chloride- and bromide-doped polypyrrole films, respectively. It is obvious that there is a difference between the fractal dimensions estimated for two different anion-doped polypyrrole films. This difference can be attributed to 3D growth of the bromide-doped polypyrrole film. An advantage of fractal analysis is its geometrical structure. In other words, this approach successfully monitored vertical difference of two surfaces. The fractal dimension of the bromide-doped polypyrrole film is slightly higher, which is due to its 3D structure. As the growth of conductive polymers can also be vertical as well as its growth across the surface, for the case of bromidedoped polypyrrole film, there was a tendency to form a 3D structure with a higher fractal dimension. Formation of ups and downs (hills and downs) is indicative of a more complicated surface in comparison with the chloride-doped polypyrrole film which has $2 \mathrm{D}$ growth across the substrate surface. It should be taken into account that this is just the difference in surface morphology as illustrated in Figures 1AI and 1BI.

However, electrochemical methods are not able to monitor fractality in nanoscale. According to the generalization made in ref 31 , electrochemical methods can detect fractal structure in the scale range 1-100 $\mu \mathrm{m}$. Therefore, the fractal analysis performed by means of electrochemical method did not detect surface morphology illustrated in Figures 1AII and 1BII to suggest this significant difference of two anion-doped polypyrrole films. Electrochemical measurements 
detected the fractal structures of the films in microscale corresponding to those illustrated in Figures 1AI and $1 \mathrm{BI}$.

We wish to inspect the surface structures of the anion-doped polymer films at different scales. In this direction, it is useful to go further to inspect the surface structure at smaller scales. None of the abovementioned techniques are capable of surface analysis at smaller scales. An appropriate technique for this purpose is SAXS, which have been commonly employed for surface analysis at scales of $0.5-200 \mathrm{~nm}$. It is also possible to estimate the fractal dimension from the SAXS data, as this approach has also been successfully applied for conductive polymers. ${ }^{19,20}$ It is well known that scattering intensity mainly includes: $:^{32}$ the diffuse scattering, and the Porod term. The last term represents the surface roughness. The Porod term is accompanied by a sharp rise of the intensity in the small-angle region, which is indicative of density fluctuations on nanoscale. The Porod term corresponds to the material structure on a scale from about 0.5 through $200 \mathrm{~nm}$. In this region, the Porod power-law suggests: $I(q) \propto q^{-\beta}$, where $\beta$ depends on the material form (e.g. mass or surface), and for fractal surfaces is equal to $6-D .{ }^{33}$ However, this relation is only valid when the scattering intensity is mainly due to the Porod term. As Ruland pointed out the validity of Porod's law should be taken into account for fractal analysis, ${ }^{34}$ and indeed an appropriate scale should be used for collecting the data.

The SAXS intensities were plotted against the $q$ factor in a logarithmic scale (Figure 3). This typical SAXS analysis corresponds to the scale of 2-20 nm. This is slightly larger than the atomic-scale, and thus, contributes the polymer chain structure. The values of the fractal dimension were estimated from curve fitting at linear region of the SAXS patterns, which

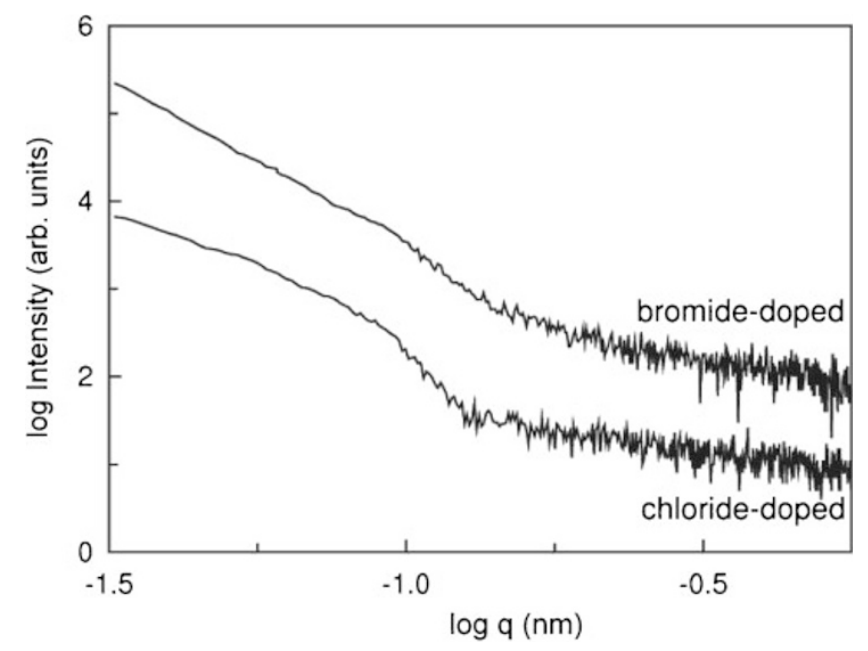

Figure 3. SAXS patterns reproduced in a logarithmic scale with respect to the $q$ factor. is indicative of a fractal power-law behavior. The SAXS analysis of the chloride- and bromide-doped polypyrrole films suggested the fractal dimensions of 2.23 and 2.38 , respectively. Although the fractal structures of the anion-doped polypyrrole film are significantly different from those at microscale, the surface fractality is still stronger for bromide doping even in nanoscale. This is indeed essential difference of microstructure and nanostructure of electrode surfaces formed in the course of electrochemical processes. ${ }^{35}$

It is obvious that a better curve-fitting is achieved for the case of bromide-doped polypyrrole film rather than that of the chloride-doped polypyrrole film. Such behavior is also observable for the fractal analysis at microscale as obtained from electrochemical measurements (Figure 2). According to the generalization made in ref 36, this is a measure of the degree of fractality. In other words, growth of polymer chain in the presence of bromide dopant is in accord with fractal pattern. On the other hand, it seems that different fractal dimensions can be detected at various scales for the case of chloride-doped polypyrrole film, as the curve slope slightly varies.

Comparison of the fractal analysis at microscale by means of cyclic voltammetry and that at nanoscale by means of SAXS indicates the significant difference of surface fractality at various scales. This is also visualized in the light of SEM imaging. The results reported here are simple but lead to an important conclusion, which is usually ignored in the literature. The difference of surface morphology of conductive polymer due to the anion-doping effect should be inspected in near-atomic scale, as large-scale (microstructure) morphology of conductive polymers is consequence of nanoscale morphology (nanostructure) of the growing polymer. In this direction, fractal analysis is an efficient tool. However, it should be employed with respect to the scale under investigation; otherwise, fractal analysis has no physical (and also geometrical) meaning. This typical study demonstrated how combination of simple investigations leads to a solid argument.

\section{CONCLUSION}

Ionic radii of doping anions have a particular influence on the surface morphology of conductive polymers. In a simple case under investigation, it was shown that morphologies of polypyrrole films synthesized in the presence of chloride or bromide ions are significantly different. This difference is more obvious in vertical morphology of the films. The other consequence of the present work is to reveal significant difference of surface morphology in microscale and nanoscale. Thus, an appropriate strategy should be 
used for fractal analysis of solid surfaces, since each technique is capable of fractal analysis at a given scale.

\section{REFERENCES}

1. L. F. Warren and D. P. Anderson, J. Electrochem. Soc., 134, 101 (1987).

2. T. Shimidzu, A. Ohtani, T. Iyoda, and K. Honda, J. Electroanal. Chem., 224, 123 (1987).

3. R. Qian and J. Qiu, Polym. J., 19, 157 (1987).

4. T. F. Otero and E. De Larreta, Synth. Met., 26, 79 (1988).

5. K. M. Cheung, D. Bloor, and G. C. Stevens, J. Mater. Sci., 25, 3814 (1990).

6. S. J. Sutton and A. S. Vaughan, Synth. Met., 58, 391 (1993).

7. C. Froeck, A. Bartl, and L. Dunsch, Electrochim. Acta, 40, 1421 (1995).

8. J. Tamm, A. Hallik, A. Alumaa, and V. Sammelselg, Electrochim. Acta, 42, 2929 (1997).

9. R. G. Compton, T. Silk, J. Tamm, and Q. Hong, Synth. Met., 93, 59 (1998).

10. C. Zhu, C. Wang, L. Yang, C. Bai, and F. Wang, Appl. Phys. A: Mater. Sci. Process., 68, 435 (1999).

11. J. Yano, F. Takamura, K. Masaoka, S. Yamasaki, Y. Ota, and A. Kitani, Synth. Met., 135-136, 417 (2003).

12. S. A. M. Refaey, Synth. Met., 140, 87 (2004).

13. M. Campos and B. Bello Jr., Synth. Met., 60, 1 (1993).

14. K. Aoki and Y. Teragishi, J. Electroanal. Chem., 441, 25 (1998).

15. Q. Hong, R. G. Compton, T. Silk, and J. Tamm, Synth. Met., 93, 65 (1998).

16. G. Merle, A. C. Grillet, J. Allemand, and D. Lesueur, Polym.
Test., 18, 217 (1999).

17. Q. Wu, L. Wu, Z. Qi, and F. Wang, Synth. Met., 105, 13 (1999).

18. J. J. Langer, Synth. Met., 113, 263 (2000).

19. S. Neves and C. Polo Fonseca, Electrochem. Commun., 3, 36 (2001).

20. C.-Q. Jin and S.-M. Park, Synth. Met., 443, 443 (2001).

21. H. Randriamahazaka, V. Noel, and C. Chevrot, J. Electroanal. Chem., 521, 107 (2002).

22. F. Gobal, K. Malek, M. G. Mahjani, M. Jafarian, and V. Safarnavadeh, Synth. Met., 108, 15 (2000).

23. A. Eftekhari, M. Kazemzad, and M. Keyanpour-Rad, Appl. Surf. Sci., 239, 311 (2005).

24. T. Pajkossy, J. Electroanal. Chem., 300, 1 (1991).

25. A. Eftekhari, Electrochim. Acta, 48, 2831 (2003).

26. T. Pajkossy and L. Nyikos, Electrochim. Acta, 34, 171 (1989).

27. A. Eftekhari, Appl. Surf. Sci., 220, 346 (2003).

28. A. Eftekhari, Colloids Surf., B, 32, 375 (2003).

29. A. Eftekhari, Appl. Surf. Sci., 227, 331 (2004).

30. M. Stromme, G. A. Niklasson, and C. G. Granqvist, Solid State Commun., 96, 151 (1995).

31. A. Imre, T. Pajkossy, and L. Nyikos, Acta Metallur. Mater., 40, 1819 (1992).

32. D. L. Williamson, Mater. Res. Soc. Symp. Proc., 377, 251 (1995).

33. K. Tanaka, A. Ito, T. Yoshii, S. Suehiro, S. Nagura, N. Ando, and Y. Hato, Carbon, 39, 1599 (2001).

34. W. Ruland, Carbon, 39, 323 (2001).

35. A. Eftekhari, M. Kazemzad, M. Keyanpour-Rad, and M. A. Bahrevar, Appl. Surf. Sci., 242, 82 (2005).

36. A. Eftekhari, J. Electrochem. Soc., 151, E291 (2004). 